\title{
Hacia un modelo de representación documental: la técnica de resumir
}

\author{
María Pinto Molina:
}

\section{RESUMEN}

Par tien do de la de fi ni ción de re su mir y de la tras cen den cia do cu men tal del re su men, se es bo zan las eta pas im pli ca das en el proceso: selección del documento, establecimientode objetivos, proceso propiamente tal, y evaluación. Se reflexiona sobre la primera fase (lectura), su importancia, tipologías y procedimientos. La segunda (análisis) está muy condicionada por la lingüística, cu yos pos tu la dos con du cen a una nue va en ti dad, el texto, como resultado de un proceso de síntesis-expansión, inverso al analítico, que transforma toda estructura conceptual profunda en su equivalente lingüística o de superficie. Se establece el concepto de macroestructura, identificable con el de resumen, y los pasos necesarios para obtenerla, teniendo en cuenta aspectos lingüísticos y metalingüísticos. La tercera fase, de síntesis o transformación, es la más delicada y creativa, se vale de dos mecanismos fundamentales: reformulación y asimilación. Se dan algunas recomendaciones sobre clarificación, reorganización y estilo, de acuerdo con criterios de fidelidad, claridad, precisión y entropía, a pesar de lo cual falta mucho para el logro de una más que necesaria Teoría de la Representación documental.

\section{ABSTRACT}

Beginning with the definition of summarization and the docu mentary im portance of summary, the implicit steps of the process are outlined: the selection of the document, the establishment of objectives, the process itself, and the evaluation. Thought is given to the first phase (reading), its importance, typologies and procedures. The second phase (analysis) is very conditioned by linguistics, whose postulates lead to a new entity, the text, as a result of a process of synthesis-expansion, the opposite of analytic process, that transforms every profound conceptual structure into its linguistic or superficial equivalent. The concept of microstructure is established, which is identifiable with that of the summary, as are the necessary steps to obtain it, keeping in mind certain linguistic and metalinguistic aspects. The third phase of synthesis or transformation, is the most delicate and creative. It consists of two fundamental mechanisms: formulation and assimilation. Certain recommendations are given regarding clarification, re-organization and style, according to criteria of faithfulness, clarity, precision and entropy. In spite of these, there is much work to do before a very needed theory of documentary representation is achieved.

\section{INTRODUCCION}

La técnica de resumir es la actividad más difícil y compleja de to das cuan tas com po nen el Análi sis Do cu men tal(AD). Su dificultad estriba precisamente en que no existen normas suficientemente válidas para aprender a realizarla. No en vano se trata de un complejo proceso que es lingüístico en la prime ra par te de su de sa rro llo, que pasa a ser conceptual en los momentos mas trascendentales, para volver a adoptar una nueva expresión lingüística en su conclusión. Precisamente por este motivo hemos de prestar una especial atención a la lin güís ti ca como dis cipli na au xi liar. En estesen ti do po de mos ca li fi car de muy pro me te do res los re cien tes hallaz gos de una joven disciplina: la lingüística textual, cuyos más destacados representantes, Van Dijk y Petöfi, pretenden conseguir una auténtica Teoría de la Representación documental, que des pe je las nu me ro sas in te rro gan tes plan tea das en la ac tua lidad en torno al proceso general de resumir.

El resultado de este complejo operativo, el resumen, es un productodocumental, creativo, polémico, y au tén tica piedra angular en el dominio de la comunicación científica. Como publicación secundaria es la más cercana al original que trata de representar, situándose en la cúspide de los productos documentales. Su presencia en el ámbito de las ciencias ha sido revolucionaria, como lo confirma el crecimiento exponencial de servicios de resúmenes. Digamos finalmente que hoy día es imposibleconcebirunprocesocientíficorazonable sin el concurso de este auxiliar ya institucionalizado a través de servicios, revistas y boletines. 


\section{EL PRO CESO DE RE SU MIR COMO UNI DAD DE AC- TUACION DOCUMENTAL}

La primerapuntualización obligada a la hora de estudiar esta actividad científico-técnica es de carácter lexicológico, pues mientras en otras operaciones se distingue terminológicamente la propia operación del producto resultante, en el caso presente y particularmente en nuestra lengua, no existe esa precisión, y un mis mo tér mi no de sig nain dis tin ta men te la actividad y el resultado. Como fór mu la es cla re ce dorahe op tado por utilizar el verbo resumir para señalar la operación, y reservar el sustantivo resumen para el producto.

Resumir es, según Mijailov, Cherniil y Guiliarevskii ${ }^{1}$ proporcionar una breve exposición del contenido de un documento. Con esta idea comulgan Van $\mathrm{Dijk}^{2}$ y Chau mier ${ }^{3}$ cuando la definen como operación por la que se abrevia el contenido de un documento y se lo representa por un cierto número de oraciones que expresan la sustancia. Fondin ${ }^{4}$ habla de "reduction" con el objetivo de analizar el contenido documental para representarloen for ma abre via da. ParaCleveland $^{5}$ es un procedimiento complejo dirigido a representar el contenido de los documentos analizados en orden a guiar al usuario en la información apropiada. Podemos concluir este breve apartado de definiciones afirmando que hay unanimidad a la hora de concebir esta operación como el pro ceso que experimentan los documentos hasta obtener la representación abreviada de su contenido.

Tras esta somera exposición introductoria, queremos se reflexione so bre la sin gu la ri dad de la ta rea de re su mir, te nien do en cuenta que en esta actividad se enfrentan (aunque distanciados) dos personajes: el autor del texto y el resumidor, con un "cam po de ba ta lla" co mún, que es el pro pio do cu mento. Las circunstancias de este especial "encuentro" son lo suficientemente singulares y complejas como para que el resumidor, úni co protagonis ta directo, tenga que poner en jue go todos sus conocimientos, destrezas y habilidades, en virtud de la multiplicidad de factores que intervienen. Se trata pues de un complejo de procedimientos intelectuales cuya individualidad está fuera de toda duda.

\section{Metodología}

Las peculiarescaracterísticas del proceso general de resumir condicionan la metodología a emplear, aunque debemos advertir que es difícil hablarde métodos en una ac ti vi dad esencialmen te in te lec tual. Im pli ca das en el proce so se en cuen tran las siguientes etapas:

- Como paso pre vio, el re su mi dor o los ser vi cios de re sú menes deberán establecer los criterios para la elección de los do cu men tos que se van a so me ter a aná li sis, ya que no todo lo que se publica es relevante y significativo, y además el cos to eco nó mi co y la dis ponibi li dad de tiem poson fac to res decisivos. No obstante, existen algunos indicadores útiles que vamos a enumerar: ${ }^{6}$
1.Razones económicas, ya que el proceso en términos de tiem po y de pro duc ción pue de ori gi nar que de una lis ta previa de do cu men tos a re su mir sean omi ti dos tra bajos de baja prioridad.

2.Naturaleza del material, su soporte, originalidad, circulación, accesibilidad, serán factores a considerar por el resumidor.

3.Origen de la publicación, en ten di do en el sen ti do de procedencia del trabajo, calidad y reconocimiento de la autoría, así como prestigio de la revista.

4.Pertinencia de los documentos escogidos, que deberán ser fuentes de información adecuadas en el dominio científico correspondiente.

5.Intereses te máticos de los usua rios, to man do en con si deración la fre cuen cia con que son so li ci ta dos de ter mi na dos documentos de un colectivo. Procedimiento cuya eficacia Lancaster pone en duda.

En definitiva, esta selección de documentos debe estar también estrechamente relacionada con la aplicación y uso del resumen correspondiente.

En segundo lugar, deberán establecerse los objetivos del análisis, delimitando el dominio científico de la actualización. Es este un dato muy importante, que no puede pasar desapercibido, pues de él dependerá el resto de la ac tua ción ana lítica, vién do se muy con di ciona do por lo que se en tien de comoentorno operativo, y que en gran medida de pen de rá del tipo de do cu men to a re su mir, de las ca racterísticas de los posibles usuarios de los resúmenes, así como las con di cio nes de tra ba jo de los re su mi do res, en espe cial las res tric cio nes que im ponen las pro pias agen cias a sus empleados.

Iniciaremos seguidamente el proceso de resumir propiamente tal, cuya exposición fragmentada es la respuesta coherenteaunos prioritarios obje ti vos ex positivos y pedagó gi cos. Para ello dis tin gui re mos tres fa ses com ple men tarias: 1 preanálisis o lectura del documento, 2 análisis o reducción, y 3 síntesis, transformación o sustitución.

Algunos autores contemplan un último paso que consiste en la evaluación del resultado obtenido, confirmando su validez a través de una serie de parámetros pre-establecidos.

\section{PREANALISIS/LECTURA/FAMILIARIZACION}

Iniciaremos este proceso con la fase de preanálisis, que haremos coincidir con la lectura del texto a resumir, correspondien do al mo men to de la fa mi lia ri za ción en tre el re su mi dor y el documento. Nuestra intención es estudiar tan solo los documentos bibliográficos que, al menos por el momento, son mayoritarios en el complejo de la producción científica, de- 
jando de un lado los no bibliográficos, aunque somos conscientes de su creciente implantación social. A partir de aquí, y como consecuencia de este singular encuentro entre lingüística y documentación, utilizaremos con frecuencia el binomio texto-documento, como objeto del estudio que nos ocupa. Con esta autolimitación de partida, podemos afirmar que la lectura es la úni ca for ma po si ble de ac ce so al contenido documental. Al respecto indica Cremmins que las habilida des y los bue nos há bi tos delectura son prerrequisitospara es cri bir bien, en cual quie ra de sus for mas, y por tan to se tie ne por vital en todo proceso resumidor. Además, y por tratarse de una operación de cambio de un lenguaje a otro, es fundamental que el resumidor domine el lenguaje del documento ori gi nal, pues de lo con tra rio, y en el me jor de los ca sos, el resumen podría es tar bien es cri to, pero no re pre sen tar el con tenido primario.

Por otra parte, y antes de seguir avanzando por el tema de la lectura, queremos se reflexione sobre el fenómeno de la escritura, para lo cual nos remitimos a los atinados planteamientos de Robert Escarpit, ' del que extraemos una idea que es fundamental para la comprensión de los fenómenos lingüísticos: y es que "el lenguaje fónico, instrumento privilegiado de la comunicación social", tiene la desgracia de que "su misma naturaleza le impide la sincronía y, en consecuen cia, la estabilidad: la fijación de la palabraen documento ha sido durante mucho tiempo un viejo sueño de la humanidad (verba volant, scripta manent)".

Para resolver problema de tal envergadura "se encontró una solución que fue puesta en marcha progresivamente en el curso de los últimos milenios. Consiste en anotar el lenguaje fó ni come dian te un len gua je vi sual de ras gos. Recordemos que no se trata en absoluto de una transcodificación, pues los mis mos ras gos cons ti tu yen sis te mas de es truc tu ras de sur perficie diferentes que corresponden a estructuras profundas paralelas, pero no idénticas, según este considerado el signo de la es cri tu ra en su va lor fó ni co o va lor vi sual". Se es ta ble ce asíla cla ra di fe ren cia en tre un len gua jeoral, que es el na tu ral por antonomasia, y otro escrito, que no deja de ser un instrumento o artificio. En cualquier caso, están en juego tres medios (palabra, escritura y rasgo) que provocan tres resultados (dis cur so, tex to eícono). Por con si guien te, es ta mos en con diciones de inferir que el texto cumple tres funciones simultáneas: discursiva, documental e icónica.

Además, "la lectura es un proceso concurrente y no simplemente simétrico de la escritura". El escritor y el resumidor, ausentes uno de otro, introducen en la relación significantesignificado connotaciones diferentes, lo que contribuye a dificultar enormemente el proceso resumidor que nos ocupa.

Para la investigadorabrasileña Cintra, ${ }^{8}$ la lectura es un proceso interactivo entre el lector y el texto que implica algo mas que el conjunto de las señales visuales escritas en este, pues depende de factores ajenos al mismo, como son el tipo de estrategias que se utilicen y el nivel de conocimientos previos que posea el resumidor. Este deberá dominar científicamente el tema a resumir, así como las técnicas generales de $\mathrm{AD}$, y mas con cre ta men te de AC. Asi mis mo, su ba ga je de cul tu rageneral tenderá a ser lo mas amplio posible, incluyendo conocimien tos so bre se miótica, ló gica, teo ría del co no ci mien to, etc.

\section{Tipos}

Tras estas consideracionesy olvidándonos de la función icónica de la lectura, que desborda nuestros objetivosmetodológicos, queremos incidir en una dicotomía que es fundamental para la correcta comprensión del proceso lector: y es el doble papel que juegan los signos gráficos, o de escritura, que por un lado son la notación codificada de un lenguaje oral, y por otro constituyen un lenguaje visual autónomo. Centrados en el lenguaje escrito, debemos destacar, por su cometido crucial en toda estructura lingüística, la palabra, concebidaporUllman" como "la más pequeña unidad de una lengua capaz de ac tuar como una ex pre sión com ple ta". Es un grafismo cerrado con estructura y rasgos pertinentes, en el que se pueden distinguir el signo fonético, o elemento fónico, y el logograma o palabra propiamente tal. De acuerdo con este planteamiento, la primera aproximación a los documentos escritos pue de hacerse asen dosniveles, provocando, en terminología de Escarpit, estos dos tipos de lectura: la hipologográfica y la hiperlogográfica. ${ }^{10}$

-En la primera lectura, el lector da al signo gráfico una respuesta estrictamente fónica, y consiguientemente "se encuentra en presencia de un discurso mutilado, sin poder beneficiarse dela memoriaexterna con te ni da en el tex to. Esta me mo ria es lo que se lla macontexto, pro duc to de la sin cronía del tex to". Ni que de cir tie ne que la fia bi li dad de esta lectura es escasa, dado el carácter ambiguo del signo gráfico, cuyaco dificación fóni caes muy im perfecta, y a ve ces, como su ce de en la len gua in gle sa, "la con ven ción so ni do/ras go no es ni estable ni sistemática". Obviamente, esta modalidad lectora interesa poco a nuestros objetivos resumidores.

-Con la lectura hiperlogográfica, en cambio, el resumidor identificadirectamenteel signográfico, efectuan dounrecorrido que "no es ya un movimiento mecánico y lineal, sino una verdadera explotación multidimensional del texto". Esta es la lectura que todo resumidor debe dominar.

En este proceso, como ya hemos mencionado, es factor de cisivo el nivel de conocimientos previos del resumidor, dato que algunos autores relacionan con la teoría de los esquemas, o especie de cuadro de referencia formado por una red multidimensional de entidades lingüísticas y conceptuales que constituyen un patrimonio personal e intransferible. Estos esquemas del resumidor se pueden aplicar a estas dos clases de lectura:

-Ladenominada botton-up (de aba jo a arri ba), que es de tipo lineal, y va de las partes al todo, es recomendable como estra te gia de lec tu ra rá pi da en la pri me ra toma de con tac to con el documento. 
-Por otra parte, la lectura top-down (de arri ba a abajo), es de carácter "explorativo", y en ella se camina a la inversa, del todo a las par tes, apro ve chan do los co no ci mien tos pre vios, o esquemas del resumidor. El analista experto no hace una lec tu ra li neal, sino que sabe leer a tra vés de lo im plí ci to y hacer predicciones sobre los enunciados siguientes. Podemos de cir pues que "la pre dic ción es un com po nen te esen cial del proce so de lec tura". Es esta varian te explorativa la que mas interesa a nuestros objetivos resumidores.

\section{Procedimientos}

En cuanto a la práctica de la lectura, digamos que esta se ve con di cio na da por múl ti ples fac to res, en tre ellos los objetivos resumidores, derivados del en tornooperativodocumental, y el tipo de documento, pues no es igual, ni tan siquiera parecido, enfrentarse a sendos textos sobre ciencias humanas y ciencias exactas. Cintra, ${ }^{11}$ distingue dos grupos de estrategias de lectura:

1. Las cognitivas, que comprenden comportamientos automáticos e inconscientes de interpretación de palabras y frases. En esta ocasión el resumidor busca las informaciones principales, procediendo a una especie de descarte de lo que es secundario. Esta estrategia de lectura se basa en los siguientes principios básicos:

-Par si monia, enun cia do por Fillmo re y Kay, me dian te el cual el lec tor (re su mi dor en nues tro caso) eli mi na de su men te lo superfluo del texto.

-Cohe ren cia, co nec ta do con las ideas de Gre i mas, Van Dijk y otros, supone la expectativa de una coherencia global y temática en el documento a leer, y viene determinada por la macroestructura.

2. Y las metacognitivas, que su po nen com por ta mien tos desautomatizados y por consiguiente una aproximación consciente al texto.

El docu men to le gible se ría aquel que exi gie se una apli ca ción equilibrada de ambos tipos de estrategias. Nótese que estas dos variedades estratégicas (cognitivas y metacognitivas) tienen una enormesimilitud con las tipologíaspareadasanteriormente esbozadas (hipologográfica e hiperlogográfica), (bottom-up y top-down).

En cualquier caso, y al margen de la estrategia adoptada, el resumidor hará una primera lectura rápida del documento original para centrar la atención en sus características fundamentales, como forma, clase, estructura de la información, etc., te nien do en cuen ta la dis tin ción, he cha por al gu nos au tores, de dos grandes categorías temáticas o argumentales: la que aglutina los temas principales, o explícitos, relacionados directamente con el contenidoexclusivo del trabajo; y la que agrupa a los secundarios o implícitos, que son tratados paralelamentepornecesidadesexpositivaso de instrumentación operativa. En cada uno de estos grupos existen subtemas, o asun tos de se gun da ca te go ría in for ma ti va y te mas aso- ciados, que ayudan al desarrollo y exposición del contenido. En esta lec tu ra, aun que su perficial, se acon se ja to mar nota de las ideas relevantes y subrayarlas en el texto cuando esto sea posible.

Pero será necesaria una segunda lectura, cuidadosayactiva, centrada en los distintos epígrafes del documento y en sus secciones claves (introducción, objetivos, metodología y conclusiones), pues por regla general contienen la esencia conceptual del documento. Este tipo de lectura es lo que Cremmins denomina“recuperativa", porque pretendeindentificar sólolos pa sajes que con ten gan in for ma ción me rece dora de ser incluida en el resumen.

A modo de conclusión, pode mos de circon Otlet, ${ }^{12}$ que el documento es un verdadero edificiointelectual, una síntesis de ideas, y no únicamente una colección ordenada de información. Y por consiguiente, la buena lectura no es un acto espontáneo. Debe ser organizada y seguir un método, pues nos encontramos ante el preludio de la producción intelectual. Se reco mien da leer len ta men te, re leer si fue ra ne ce sa rio, leer tomando notas y, a veces, leer en voz alta, teniendo siempre muy en cuenta el error que conlleva el preconcepto bibliotecario de lectura "única y absoluta". Por el contrario, hay una su per po si ción de las ideo lo gías del au tor del do cu men to y del resumidor. Como afirma Cunha" "negar el componente ideológico en el análisis del texto para fines documentales es no saber situar el papel del individuo en los procesos de producción-consumo". Al mismo tiempo sería negar el carácter social del lenguaje.

\section{ANALISIS/EXTRACCION/REDUCCION}

Pero la lec tu ra no se jus ti fi ca por sí mis ma, sien do tan solo un medio de aprehensión textual en pos de unos objetivos. Por ello, una vez finalizada esta etapa, que culmina con la asimilación de los conocimientos aportados por el texto, y que es equiparable a la toma de datos de cualquier trabajo intelectual, el resumidor acometerá el análisis propiamente tal, comenzando con la segmentación-selección de las palabras, frases y párrafos representativos del documento.

\section{Condicionantes teóricos}

Llegamos así a la fase de explotación y aprovechamientodel material a analizar, que representa sin duda alguna el momento más difícil y controvertido de todo el proceso. En el desarrollo de este trabajo se lec ti vo, y pues to que aún nos movemos en el dominio del lenguaje, adquiere un especial prota go nis mo la lin güís ti ca, si tua da en la base de cien cias tan antiguas como la lógica o la propia matemática. Anotemos sin embargo, que aunque totalmente imprescindible, no deja de ser un instrumento o intermediario que, mediante el establecimiento de un código, el lenguaje, posibilita la comunicación documental entre los individuos.

Pero el con te ni do de los do cu men tos, obje tivo del procesoresumidor que nos ocupa, es de carácter conceptual, y como tal desborda los estrictos cauces de la lengua para inundar el 
infinito uni ver so de los con cep tos. Con vie ne pues es table cer, en el eje discursivo de todo texto-documento, la distinción entre dos grandes planos: el básico, o conceptual; y el auxiliar, o lingüístico.

\section{Aspectos lingüísticos}

Tras esta necesaria aclaración de partida, queremos insistir sobre los aspectos lingüísticos, apoyándonos en las especiales características de la célulabásica de toda lengua, el signo lingüístico. Este, como realidad dual significante-significado, se nos presenta bajo dos aspectos claramente diferenciados: el morfológico, en el plano signficante o de la expresión; y el sintáctico-semántico, en el plano del significado o contenido, que es el interesante para el resumidor.

Por otra parte, el signo se mueve según dos ejes: el sintagmático, o combinatorio, que contempla sus posibles agrupamien tos; y el paradigmático, o se lec ti vo, que re fle ja las re laciones existentes entre los signos capaces de asumir una misma función. Estos dos ejes son virtuales, y determinan la organización general del discurso. En el caso del proceso resumidor, cuyo objeto son los textos, el reconocimiento de las relaciones sintagmáticas y paradigmáticas nos lleva a la formulación de los campos semánticos, que posibilitan una mejor organización de los procedimientos de análisis, así como la construcción de instrumentos y productos. Además, a través de es tas re la cio nes se hace po si ble la re u nión de uni da des lé xi cas en gru pos es truc tu ra dos, de modo que cada uni dad se defina por el lugar que ocupa con respecto a las demás.

Todo ello nos permite afir mar, en lí neas ge ne ra les, que elestructuralismo resulta muy eficaz en el estudio del eje paradigmático, en tanto que el transformacionalismo, también llamado generativismo, y el distribucionalismo, prestarán grandes aportaciones en el eje sintagmático o combinatorio, y en el plano del contenido,inten tan doex plicara tra vés de la gramática generativa, la estructura lógica del pensamiento que suministra sentido a los discursos.

Ambas corrientes, que son complementarias, interesan a nuestros objetivos resumidores, y aunque ninguna de ellas satisface plenamente los objetivos de AD de contenido, si que podemos afirmar que la utilización conjunta de ambas permite una aproximación analítica global y satisfactoria.

Además, y puesto que el problema resumidor lo hemos centra do en la trans for ma ción de una ex pre sión lin güís ti ca en un contenido conceptual, el resumidor debe ser consciente en todo momento de la amplitud y complejidad del concepto de significado o contenido, circunstancia que desborda los estrictos cauces de la lingüística y nos remite, es el caso de la semántica cognitiva, al conocimiento que del "mundo" tienen el autor del documento y el resumidor, lo que anteriormente y con mo ti vo de la lec tu ra ha bía mos de no mi na do "esquemas". Todo ello nos confirma que es difícil dominar un tema como el del significado, que "con cier ne a mu chas ciencias sociales y no es abarca do en su to ta li dad por una sola de ellas". ${ }^{14}$
Tampoco se podrá olvidar la realidad más amplia que lo englo ba y con di cio na. Se tra ta del contexto, uno de los factores de la ac ti vi dad lin güís tica mas ne ce sario para la ex pli citación de los mensajes. Como afirma Lyons, "el significado del enunciado depende fundamentalmente del contexto". Este puede ser explícito, o lingüístico; o bien implícito, y en este caso es ca li fi ca do deextralingüístico o situacional, tam bién muy aprovechable para la interpretación semántica que vamos buscando.

En cuan to a las di men sio nes del sig no lin güís ti co, con vie ne a nuestros objetivos resumidores distinguir tres estadios perfectamentedelimitados: la palabra, o unidad míni ma de significación; el enunciado, o nivel mínimo de enunciación; y el texto, o unidad intencional de comunicación cerrada.

La palabra es la célula base de toda estructura de lenguaje, conformando lo que algunos expertos denominan el plano funcional o selectivo, en el eje paradigmático. Se la vincula al léxico, y también al clásico concepto de diccionario, permitiendo el tipo de análisis conocido como componencial. Se pueden distinguir dos categorías de palabras: las útiles, impropiamente llamadas palabras vacías, que expresan relaciones sin tác ti cas u ope ra to rias; y las nocionales o informativas, que se subdividen en tres subcateogrías: generales, circunstanciales y específicas. En general, y salvo el caso de las palabras útiles, cuya función está claramente definida por la gra má ti ca, la fun ción de una pa la bra no pue de per ci birse más que en el funcionamiento del texto.

El enunciado o frase, se constituye el eje sintagmático, o combinatorio, de la lengua. Su significado es lo que conocemos como proposición, punto de partida obligado en todo análi sis de con te ni do. Has ta fe chas no muy le ja nas, la fra se u oración ha satisfecho plenamente los objetivos investigadores de los lingüistas.

No obstante, los recientes estudios sobre el texto han venido a ensombrecer esta supremacia frásica, en beneficio de una nueva y pujante disciplina, la gramática textual, también conocida como lingüística del texto, especialidad que nace con vocación de interdisciplinar e integrar. Surge así el concepto de secuencia de fra ses, como con jun to de es tas que reunen unas mínimas condiciones de conexión y coherencia. Pero no todas las secuencias de frases constituyen un texto, pues este exi ge ade más, y como re qui si to es pe cífico, la coherencia global. ${ }^{16}$

Ya en el terreno de los mecanismos lingüísticos, anotemos, como in di can Gre i mas y Petöfi, ${ }^{17}$ que la primerarealidad lingüística del texto es la de su composición, esto es, la de su producción como síntesis, siendo derivado o secundario lo que no so tros pre ten de mos ha cer, en el ca mi no in ver so, es decir el estudio de los mecanismos analíticos desde la base del pro duc to tex tual aca ba do. Son pues dos ope ra cio nes de sen tido contrario que fundamentan los dos grandes sectores de la teoría textual. 
La síntesis, por su parte, está directamente relacionada con una de las propiedades específicas de las lenguas naturales, como es la elasticidad del discurso. Algunos autores, entre ellos Petöfi, uno de los mas destacados pro mo to res de la gramática textual, nos hablan de un principio que consideramos fundamental para la comprensión del ciclo síntesis-análisis que pretendemos exponer: es el principio de isomorfía lingüística, se gún el cual la or ga ni za ción de nues tros pro duc tos humanoscomunicativo verbales se realiza de acuer do con un proceso de expansióncuantitativa que, sin embargo, respeta la célula lingüística elemental.

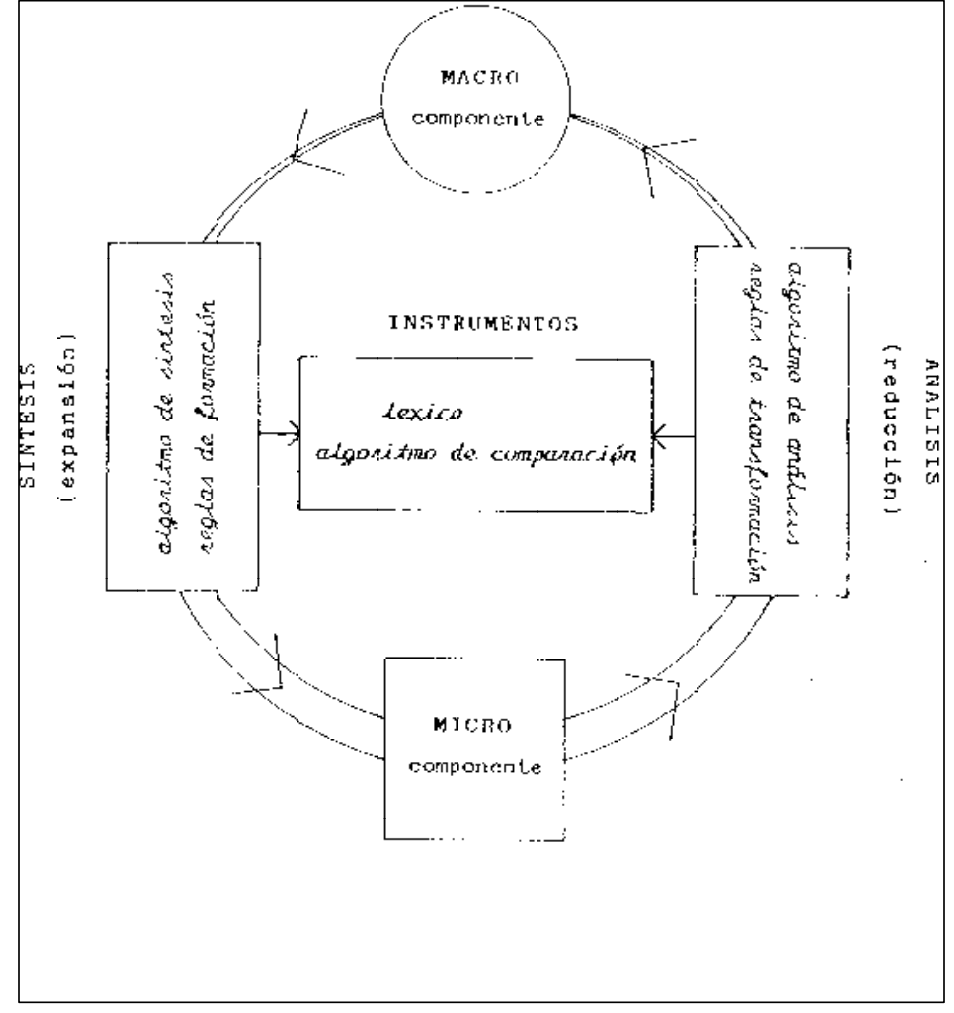

Este proceso de expansión, en terminología de Greimas, o generación, se gún Petöfi, su je to a una se rie de re glas es pe cíficas, desemboca en la escritura del texto. Centrados en este, dis tin guen los es tu dio sos, a la luz de las apor ta cio nes ge ne rativistas, dos tipos de estructura:

* La de superficie, que es de naturaleza antropomorfa, y se corresponde con las últimas etapas transformativas inmediatas a la manifestación lineal del documento.

* Y la profunda, también llamada por Pottier estructura de entendimiento,${ }^{18}$ completamente inasequible a la observación inmediata, pues se mueve en el plano conceptual.

Además, no se trata de estructuras independientes. Por el contrario, la relación entre ambas es directa e inmediata, ya que la estructura profunda es generadora de la superficial, mediante unos complejos procesos de transformación. Por otra parte, las trans for ma ciones sin tác ticas no aportan, se gún Chomsky, nin gún su ple men to de sig ni fi ca ción; y en este sentido podemos afirmar que toda forma de superficie tiene su forma profunda equivalente. De cara a nuestros objetivos resumidores nos hallamos ante un trascendental descubrimien to. Del he cho de que am bas es truc tu ras tex tua les (su perficial y profunda), cuya diferencia en tamaño o extensión es considerable, sean conceptualmente equivalentes se desprende nuestro interés metodológico por descubrir la estructura profunda en virtud de un principio elemental de economía textual o entropía.

Llegamos de este modo al concepto de macroestructura, equiparable al de estructura profunda aquí esbozado, como desarrollo transfor mati vo, je rarquiza doy coherente de un tópico inicial, o tema principal del documento. La macroestructura contiene pues toda la información esencial del texto desarrollado. Según Van Dijk, es una "representación abs trac ta de la es truc tu ra glo bal de sig ni fica do de un tex to". De acuerdo con este planteamiento, no resulta aventurado anticipar la sinonimia existente entre resumir un texto y obtener su macro-estructura.

Pero el concepto de macroestructura se nos ofrece como relativo, ya que siem pre se re fie re a otras es truc tu ras más específicas de un nivel inferior. De ello se deduce que existen dis tin tas ca te go rías en un mis mo tex to, ya que cada ni vel "sur perior" de proposiciones pue de re presen taruna macroes tructu ra fren te a un ni vel in fe rior. Es de cir, que en tre la es truc tu ra superficial y la profunda exis ten una se rie de es ta dios in termedios de acuerdo con una disposición arbórea. La macroestructura del texto, la que interesa a nuestros objetivos resumidores, es la más general y global de todas ellas.

No obstante, y a pesar de los avances espectaculares experimentados en este dominio, debemos reconocer que, en el esta do ac tual de pro gre sos de la lin güís tica, "eles ta ble ci mien to de unas reglas constitutivas y, sobre todo, transformativasde la estructura profunda textual es mas bien un desideratum que una realidad". ${ }^{20}$

En la actualidad, los esfuerzos in vestigadores se dirigen aestudiar los me canis mosque con du cen des de un es ta do de macro- estructura textual al de micro-estructura correspondien te, proce so que es ex pan si voy sin te ti za dor, y que su po ne como ya se ha indicado el recorrido inverso al preceptivo en todo aná lisis de contenido, pues este debe iniciarse en la superficie (micro- estructura) para alcanzar las profundidades del contenido documental (macro-estructura).

Teóricamente, y acorde con las consideraciones lingüísticas ex pues tas, en esta eta pa ana lítica el do cu men to ori gi nal se ve sometido a un proceso que constará al menos de los siguientes pasos:

- En primer lugar, y por medio de una serie de transformaciones que Pottiercalifica de externas, con ver sión del texto de par ti da, cuya con fi gu ra ción esmorfo-fonológica, en estructuras léxico-sintácticas. Es este un imprescindible paso intermedio.

- En segundo lugar, será necesaria una transformación que ya es interna, de las estructuras léxico-sintácticas en re- 
presentaciones lógico-semánticas, en el caminodeacceso al contenido de los documentos, que es nuestro objetivo prioritario.

- Faltaría un tercer paso, el más importante, que, mediante una transformación generativa, nos condujese desde estas representaciones lógico-semánticas hasta las llamadas estructuras deentendimien to, o macroes tructuras, tras pasando el umbral de la lingüística y desembocando en el plano de los conceptos. Es lo que algunos lingüistas denominan conceptualización, origen y destino de toda comunicación lingüística.

De una manerabas tan te simplificada, aun que in te re san te por motivos metodológicos, podemos esquematizar el camino analítico en el gráfico siguiente.



\section{Aspectos metalingüísticos}

Si bien la pri me ra ope ra ción, que con vier te el tex to en un sistemadees truc turas léxico-sintácticas, es relativamentefácil, pues está perfectamente reglamentada por la lingúística, ya que la manifestación li neal y la sin tác ti ca son su ma men te estables y tienen una combinatoria muy estricta, no sucede igual con la se gun da, que pre ten de con du cir nos a la re pre sentación ló gi co-se mán ti ca del dis cur so, pues el cam po de la semántica es relativamente abierto y posee una enorme maleabilidad combinatoria. Además, como apunta Ullman, "' "el referente, rasgooacon tecimien to que da fue ra de la pro vincia lingüística".

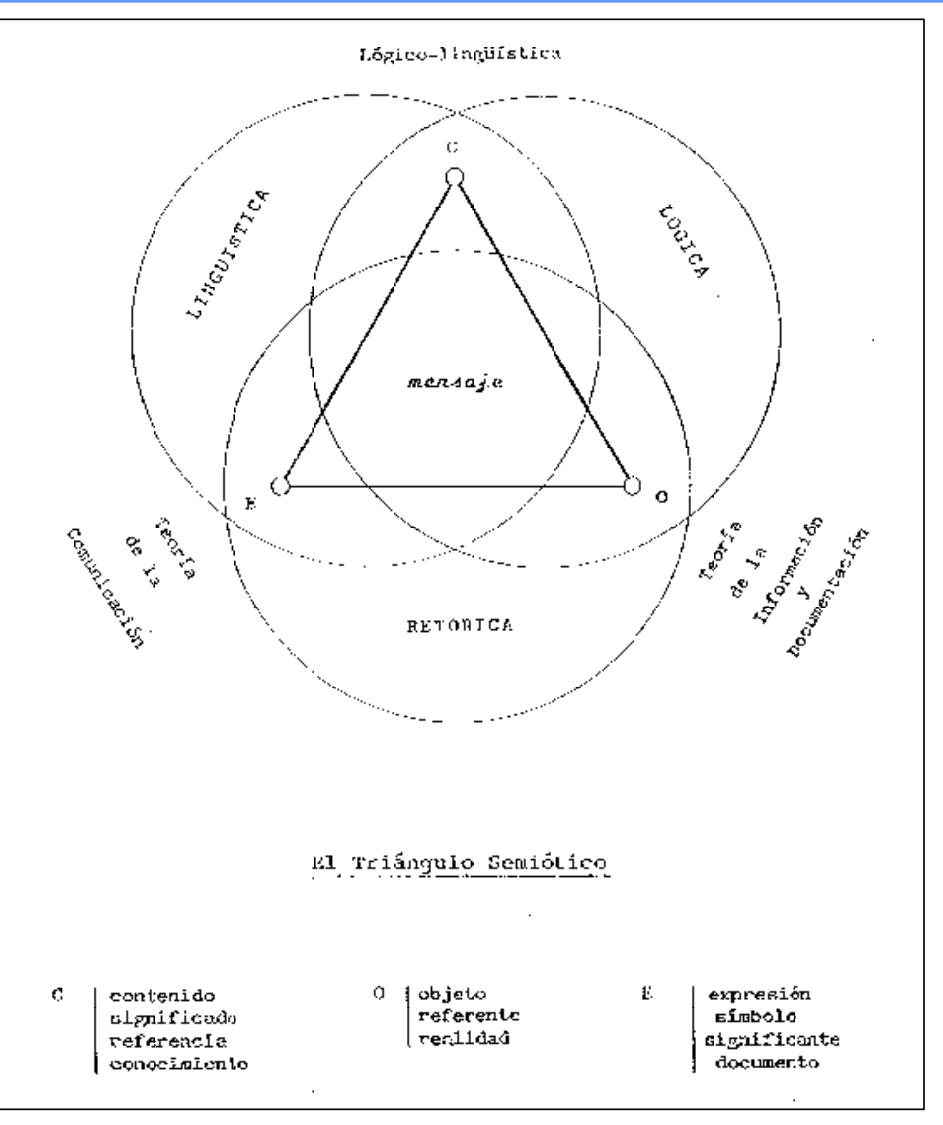

Lo que acabamos de indicar se muestra claramente en el ya famoso triángulo de Ogden y Richards. Como puede observarse en este esquema, el referente (también denominado ob je to o rea li dad) se en cuen tra en el te rri to rio de lalógica. La utilización del sentido común por parte del resumidor implica una serie de procedimientos lógicos, que se resumen en la identificación y en la estructuración de las llamadas informaciones "significativas" o "relevantes". Se tendrán en cuenta las dos vertientes de la lógica, la general y la formal. Por me dio de la ló gi ca general, y prin ci pal men te en lo que se refiere al discurso en ciencias humanas, se examinarán los proce sos de ad qui si ción de los co no ci mien tos cien tífi cos, así como la cons truc ción de los prin ci pios, hi pó te sis, le yes ge nerales y teorías. La lógica formal nos explicará como se elaboran los raciocinios desde el punto de vista formal. A este res pec to es con ve nien te in di car que la cien ti fi ci dad de un discurso depende de la articulación entre las exigencias de ambos modelos de lógica.

Además, este apoyo inexcusable en la lógica, obliga al resumidor a tomar contacto con el amplio universo de la ciencia, y más concretamente con las ciencias sociales y del pensamiento, como la psicología, la sociología, la psicología social, la teoría de la comuni ca ción, la teo ría de la in for ma ción, la informática, la filosofía, la ideología..., e incluso, aunque indirectamente, la matemática.

\section{Procedimientos}

Y de los as pec tos teó ri cos fun da men ta les, pa se mos a la rea lidad operativa más inmediata. 
Segmentación y selección

Con los datos suministrados por las diferentes lecturas, podremos acometer el auténtico análisis del documento, comen zan do con una se rie de pa sos pre vios. El pri me ro de ellos es la segmentación, tarea empírica que apunta a descomponer provisionalmente el texto en magnitudes más manejables, mediante la división en segmentos o unidades sintagmáticas provisionales.

Todo ello conlleva un desmenuzamiento de la información explorando las partículas más pequeñas hasta que las partes pertinentes de la estructura queden expuestas y dispuestas para ser escritas. Es esta una necesaria y difícil labor de "striptease" que nos permitirá ver al desnudo las auténticas apor ta cio nes del do cu men to en es tu dio, eli mi nan do, en el período de selección que sigue, toda la informaciónsecundaria o irrelevante.

Digamos al respecto que aunque no resulta fácil definir en qué consiste la comprensión de un texto-documento, si que se sabe que esta no es lineal. Como señala Pottier, ${ }^{22}$ se conceptualizan segmentos del discurso constantemente remodelados por la conceptualización de los segmentos siguientes. Se transforma sin parar lo semántico en conceptual.

\section{Identificación}

Llegamos así al momen to de iden tificar las partes sig nificativas del documento, para lo cual es obligadoque ten gamos en cuenta las enormes diferencias formales existentes entre los distintos discursos científicos. Esta realidad nos conduce al conepto de superestructura textual, concebida por Van Dijk $^{23}$ como una es pe cie de es que ma al que el tex to se adap ta, con independencia de su contenido, permitiendo establecer una determinada tipología textual. A grandes rasgos, y por tratarse de las superestructuras más habituales, hablaremos aquí de las narrativas y de las argumentativas.

Los textos de superestructura narrativa, importantes en la comunicación textual, obedecen a un esquema basado en la secuencia trama-resolución.

Por su parte, las superestructuras argumentativas se basan en lasecuen cia hipótesis-conclusión,encontrándose entre ellas el tratado científico, al que por razones obvias vamos a dedicar mayor atención, destacando sus indicadores básicos, que pueden esquematizarse del modo siguiente: ${ }^{24}$

1.Objetivos y alcance: ya que el resumidor deberá cuestionar se por qué se es cribió el docu men to, y qué pro pó si to tuvo su autor. De esta manera podrá comprender la naturaleza del problema y el alcance de la investigación.

2.Metodología: re co gien do, si secon si de racon venien te para la comprensión, las técnicas y métodos usados por el autor del documento ori gi nal; y re sal tan do, so bre todo en los trabajos experimentales, los nuevos métodos, equipos y material empleados. En los no experimentales, se anotarán las fuen tes de los da tos y cómo han sido ma ne ja dos y pre sentados en el texto.

3.Resultados: que deben ser presentados de forma clara, recogiendo los descubrimientos de manera concisa aunque in for ma tiva. La ma yo ría de los teó ri cos dis tin guen en tre resultados y conclusiones, basándose en el clásico procedimiento experimental en el que el experimentador asegura los hallazgos y luego procede a interpretarlos.

4.Conclusiones: que describirán las implicaciones de los resultados y especialmente cómo éstos se relacionan con el propósitodelainvestigación.Pueden serrecomendaciones, sugerencias, aplicaciones, nuevas relaciones, hipótesis,... En definitiva, son el con cen tra do o esen cia de la in ves ti gación, y por con si guien te de gran va lor e in te rés en el pro ce so resumidor.

\section{Algoritmo de análisis}

Superados estos pasos previos, digamos que en esta etapa analítica, desempeñan un papel fundamental las reglas de transformación ya mencionadas, muy similares a las reglas de la génesis sen tencialde la gra mática transformati va generativa. ${ }^{25} \mathrm{Se}$ basan en los modelos más prestigiosos de la descripción lingüística, y obedecen al anteriormente aludido principio de la isomorfía lingüística. Puesto que se trata de una se rie or de na da de ope ra cio nes que per mi ten el paso de un estado inicial microestructural a su correspondiente estado fi nal de ma croes truc tra, po de mos ase gu rar qe esta se rie de reglas constituyen un auténtico algoritmo de transformación sus cep ti ble de ser au to ma ti za do en un fu tu ro no muy le ja no.

Van Dijk ${ }^{26}$ las denomina macrorreglas, como instrumento que posibilita la unión entre micro y macroestructuras. Son operaciones tendentes a la reducción de información semánti ca, y se apli can a la se rie de pro po si cio nes que con for man el texto para obtener su macroestructura general. Las principales, o básicas, son cuatro: dos de anulación, omisión y selección, y dos de sustitución, generalización e integración.

La primera macrorregla, OMITIR, resulta bastante trivial y significa que toda información de poca importancia y no esencial puede ser omitida. Mediante la macrorregla de SELECCIONAR se excluyen las proposiciones que son condiciones, parte integrante, presuposiciones o consecuencias de la proposición no omitida.

Estas dos macrorreglas de anulación, se pueden equiparar con lo que Es car pit ${ }^{27}$ denomina contracción, en ten di dacomo la eliminación de la redundancia textual, que, en nuestra lengua escrita, puede oscilar en torno al 50\%. Por consiguiente, todo texto puede ser re du ci do a la mi tad, sin me noscabo de su información significativa.

Por otra parte, y en relación con las macrorreglas de sustitución, digamos que al GENERALIZAR, variasproposiciones relacionadas entre sí se sustituyen por una sola proposición 
representativa de todas ellas.

La cuarta regla, CONSTRUIR o INTEGRAR, sustituye una serie proposicional por una nue va pro posición que presu pone a todas las de la serie.

En el plano operativo, esta reducción de los componentes menos esenciales del significadodel texto, se efectúateniendo en cuenta los siguientes consejos: ${ }^{28}$

1. Indique el resultado y omita como se consigue.

2. Para hechos conocidos, evite ejemplos, definiciones y explicaciones o especificaciones adicionales.

3. Elimine las proposiciones con poco contenido.

4. Elimine los gráficos, tablas y referencias.

5. Omita aquello que el autor juzgue menos importante.

6. Pres cin da de lo que re sul te ob vio, así como de pun tos y argumentos marginales.

7. Sea po si ti vo, pres cin da de lo que no se haya di cho o es crito.

8. Tome la clase, grupo o conjunto, no sus miembros.

Lle ga do a este pun to, y de bi do a los fac to res ex tra lin güís ticos que intervienen en la operación de resumir, debo aclarar que la macroestructura es subjetivamente variable, en función de las creencias ("mundo" o "esquemas") evaluadoras del resumidor, lo que representa una limitación importante en la apli ca ción de lasmacrorreglas que, "pese a te ner un ca rác ter general como principios de organización y reducción global de la información, pueden aplicarse de diferentes maneras para distintos tipos de texto en distintos contextos pragmáticos". ${ }^{29}$ Por consiguiente, la formación sistemática de una teo ría de la ma croes truc tu ra de pen de rá no sólo del pro fundo es tu dio del tex to, sino tam bién de su superestructura, del contexto en que se encuentre y de los mencionados esquemas.

\section{TRANSFORMACION/REPRESENTACION/ SUSTITUCION}

Como fase final está la síntesis, o arte de componer la información resultante del análi sis. Es sin duda al gu na el mo mento más de li ca do y di fí cil. Si has ta aquílas ac ti vi da des ana líticas se han podido someter a unas técnicas más o menos afor tu na das, es prác ti men te im po sible es table cer unos me canismos sintetizadores que sean válidos para todo tipo de documentos y de resumidores. Nos encontramos ante la tarea suprema, exclusiva del resumidor, donde éste debará ponder en juego sus cualidades, habilidades y conocimientos para recrear el documento original a escala reducida. Esta fase es, se gún al gu nos, la del re su mir pro pia men te tal. Pero el proceso, globalmente considerado, es de carácter analítico, a pesar de que esta última y trascendental fase sintetizadora haya llevado a algunos a la errónea conclusión de que resumir equivale a sintetizar.

\section{Estrategias}

Esta última y definitiva etapa sintetizadora se corresponde pues con la transformación de las macroestructuras derivadas del proceso analítico hasta obtener la macroestrucrtura general del texto-documento.

Esta transformación se realiza a través de dos mecanismos fundamentales:

- La reformulación, mediante la cual se incorpora una macroestructura a un grupo de ellas ya existente.

- La asimilación, cuando determinadas macroestructuras se unen a través de una operación de síntesis.

De este modo, y tras la aplicación reiteradadees tos mecanismos, llegaremos al producto final o resumen, que se establece como documento secundario autónomo, esto es, un texto breve y completo gramaticalmente, que recoge el contenido esencial del documento original. Su mensaje tiene significación e im por tan cia por sí mis mo, pues sus con te ni dos pue den ser entendidos sin necesidad de recurrir al documento original. ${ }^{30}$ Pero sin duda al gu na, esta eta pa acu sa más que las an terio res la apor ta ción del re su mi dor, ya que de ma ne ra de fi ni tiva debe presentar la macroestructura general de un documento con unas proposiciones de nueva elaboración, pero sin alterar en nada su mensaje, es decir sin introducir elemento semántico alguno que no contuviese el original.

\section{Recomendaciones generales}

Las consideraciones prácticas para la síntesis son encaminadas hacia estos tres aspectos:

\section{A) Clarificación:}

1. Busque expresiones precisas.

2. Defina el concepto central si fuera necesario para su comprensión.

3. Si no encuentra una formulación clara, elimínela.

4. Si un acró ni mo noes co mún, es pe ci fí que loy acláre lo cuando aparezca por primera vez.

5. Use terminología normalizada.

\section{B) Reorganización:}

1. Elimine proposiciones sin referencia.

2. Reajuste la coherencia y estructura textual. 
Elección de do-



Preparación del material


Omitir Seleccionar Generalizar Integrar

SINTESIS

Reformulación y reorganización de resultados

Representación

Representación

Macroestructuras

- de los resultados

RESUMEN

Consideraciones estilísticas

EL PROCESO GENERAL DE RESUMIR:

Metodología

3. Sitúe la oración tópica en la posición principal.

4. Reajuste la estructura de la oración.

5. Ponga las afirmaciones análogas juntas.

\section{C) Estilo:}

1. Use tiempo presente y formas activas de verbos.

2. Use conectores de oraciones.
3. Elimine construcciones toscas y expresiones retóricas.

4. Utilice frases cortas.

De acuer do con Bo ret y Peyrot, ${ }^{31}$ la ela bo ra ción de todo re sumen estará precedida por los siguientes criterios:

1. Fidelidadaloriginal, que deberá ser respetado en su conte ni do, sin omi sión de par tes sus tan ciales, evi tan doel resumidor cualquier apreciación personal. 
2. Precisión, con el empleo de términos justos, eludiendo la redundancia y la repetición.

3. Claridadexpositiva, utilizandolaterminología apropiada a cada documento. Eles tilo debesermusculoso, nervioso, sin tejido conjuntivo.

4. Entropía, dan do a la fra se la ple ni tud de sen ti do con el míni mo de pa la bras, re du cien do los cos tos de edi ción y al macenamiento en memorias masivas.

Como es fácil comprender, una de las dificultades de la ope- ración de resumir surge a la hora de compatibilizar estos criterios, pues algunos son antitéticos. El éxito estribará en el logro de un equilibrio satisfactorio.

\section{Epílogo}

Digamos finalmente que falta mucho para definir un modelo de ope ra ción de re su mir con el de sea ble gra do de pre ci sión y fiabilidad. En este artículo hemos tratado de esbozar los primeros pasos hacia tal modelo empírico, que proporcione a la Do cu men ta ción una am plia y prác ti ca ex pli ca ción de la Teoría de la Representación de documentos en lo que a su contenido se refiere.

\section{NOTAS BIBLIOGRAFICAS}

1. Mijailov, A.I.; Chernii, A.I.; Guiliarevskii, R.S.: FundamentosdeInformática. La Habana, Academia de Ciencias de Cuba, 1973, v.I., p. 212.

2. Dijk, M. V., Slype, G.:Elserviciode Documentaciónfrente alaexplo sióndelainformación. Buenos Aires, Consejo Nacional de Investigaciones Científicas y Técnicas, 1972, p. 22.

3. Chaumier, J.: Análisis y Lenguajes documentales. Barcelona, Mitre, 1986, p. 22.

4. Fon din, H.: "La struc tu re et le vocabu lai re del'a naly se docu men taire". Documentaliste, v.14, no. 2, 1977, p. 13 .

5. Cle ve land, D. B.: Introduction to indexing and abstracting. Little ton, Libraries Unli mited, 1983, p. 104.

6. Maizell, R.: Abstracting scientific and technical literature. Nueva York, Wiley Interscience, 1971, p. 35.

7. Escarpit, R.: Teoríage ne ralde la información yde la co municación. Barcelona, Icaria, 1981, p. 162.

8. Cin tra, A.M.: "Es tra te gias de lei tu ra en do cu men taçao". En:Análisis Documentaria: aanáliseda síntese". Grupo Temma. Brasilia, MCT, 1987, p.28.

9. Ull mann, S.:Semántica: introducciónalacienciadelsignificado. Ma drid, Agui lar, 1980, p. 36.

10. Escarpit, R.: Op. cit., p. 167.

11. Cintra, A. M.: Op. cit., p. 32

12. Otlet, P.: Traité de Documentation... Bruselas, Mundaneum, 1934, p. 317

13. Cun ha, I. F.:Análisedocumentaria: aanálisede sintese. Gru po Temma. Bra si lia, MCT, 1987, p. 53

14. Lyons, J.: Lenguaje, signficado y contexto. Barcelona, Paidós, 1983, p. 23.

15. Ibid., p. 197.

16. Dijk, T. Van: La ciencia del texto. Barcelona, Paidós, 1983, p. 55 y ss. 
17. Petöfi, J., García Berrio, A.: Lingüística del texto. Madrid, Alberto Corazón, 1979, p. 72

Greimas, A.J.: Semántica estructural. Madrid, Gredos, 1987, p. 250.

18. Pottier, B.: Lingüística general: teoría y descripción. Madrid, Gredos, 1987, p. 14

19. Dijk, T. Van: Op. cit., p. 55

20. Petöfi, J. S.; García Berrio, A.: Op. cit., p. 66

21. Ull man, S.: Semántica: Introducciónalacienciadelsignficado. Madrid, Agui lar, 1980, p. 64

22. Pottier, B.: Op. cit., p. 32

23. Dijk, T. Van: Op. cit., p. 141 y ss.

24. So bre este parti cu lar sirva de base los es tu dios de Cle ve land, A.\& D.: In troduction to in dexing and abastracting. Litlle ton, Li bra ries Un li mi ted, 1983, p. 112-119; y Borko, H., Chat man, S.: Criteria for acceptable abstracts: a survey of abstracters'instructions. American Documentation, april 1963, p. 151.

25. Petöfi, J., Garcia Berrio, A.: Op. cit., p. 68 y ss.

26. Dijk, T. Van: Op. cit., p. 59. Texto y contexto. Madrid, Cátedra, 1980, p. 204 y ss.

27. Escarpit, R.: Teoría general de la información y de la comunicación. Barcelona, Icariz 1981, p. 212.

28. En ders- Nigger me yer, B.: "Con tent analysis: a spe cial case of text com prehen sion". En: Information-Knowledge-Evolution. Ed. S. Koskiala y R. Launo. Proceedings of the 44 FID Congress. North Holland, 1989,p. 110.

29. Dijk, T. Van: Op. cit., p. 64

30. Moreiro González, J.A.: "El resumen científico en el contexto de la teoría de la documentación. Tex to y des crip ción sus tan cial”. Do cumen ta ción de las Ciencias de la Informa ción, No. 12, 1989, p. 156.

31. Boret, M., Peyrot, J.: Le resumé de texte. Paris, PUF, 1969, p. 31 